\title{
Properties of Electrons Scattered by a Strong Plane Electromagnetic Wave with a Linear Polarization: Semiclassical Treatment ${ }^{\text {TI }}$
}

\author{
O. V. Bogdanov ${ }^{a}$ and P. O. Kazinskib,* \\ ${ }^{a}$ Department of Higher Mathematics and Mathematical Physics, Tomsk Polytechnic University, Tomsk, 634050 Russia \\ ${ }^{b}$ Faculty of Physics, Tomsk State University, Tomsk, 634050 Russia \\ *e-mail:kpo@phys.tsu.ru \\ Received November 11, 2014
}

\begin{abstract}
The problem of scattering of ultrarelativistic electrons by a strong plane electromagnetic wave of a low (optical) frequency and linear polarization is solved in the semiclassical approximation, when the electron wave packet size is much smaller than the wavelength of electromagnetic wave. The exit momenta of ultrarelativistic electrons scattered are found using the exact solutions to the equations of motion with radiation reaction included (the Landau-Lifshitz equation). It is found that the momentum components of electrons traversed the electromagnetic wave depend weakly on the initial values of momenta. These electrons are mostly scattered at small angles to the propagation direction of the electromagnetic wave. The maximum Lorentz factor of electrons crossed the electromagnetic wave is proportional to the work done by the electromagnetic field and is independent of the initial momentum. The momentum component parallel to the electric field vector of the electromagnetic wave is determined solely by the laser beam diameter measured in the units of the classical electron radius. As for the reflected electrons, they for the most part lose the energy, but remain relativistic. A reflection law that relates the incident and reflection angles and is independent of any parameters is found.
\end{abstract}

DOI: $10.1134 / \mathrm{S} 0021364015030030$

\section{INTRODUCTION}

The scattering of electrons by the electromagnetic wave is a textbook subject both at classical and quantum level. Even in the regime of strong strengths, the transition amplitude in a one-vertex approximation is well-known and calculated using the exact solutions to the Dirac equation (see, e.g., [1]). However, for the laser radiation with large intensity (it should be of the order of $I \geq 10^{24} \mathrm{~W} / \mathrm{cm}^{2}$ for the optical laser), the process of radiation of soft photons becomes relevant and even dominates at certain circumstances. Hence, an infinite number of diagrams have to be summed in order to describe correctly the properties of the scattered electrons and photons in this regime. Furthermore, at such intensities, the scattering amplitudes depend nonlinearly on the field strength of the electromagnetic wave [2] and so the results strongly depend on the shape of the laser beam. The lasers with such intensities will become accessible in the nearest future $[3,4]$, but a thorough theory of interaction of strong electromagnetic waves with a matter is far from being complete. We report on new (to our knowledge) startling features of the unpolarized electrons scattered by a laser beam with linear polarization. These properties, in particular, allow one to manipulate the characteristics of electron bunches, in addition to the standard means (see, e.g., [5]).

\footnotetext{
"The article is published in the original.
}

The issue of direct summing an infinite number of diagrams with soft photons and dressed electron propagators is a formidable task. Therefore, we shall study this problem in the semiclassical approximation neglecting the radiation of hard photons with the energies comparable to the electron energy, the vacuum polarization effects, and the electron-positron pairs production processes. This is a reasonable approximation for the electromagnetic field strengths less than the critical (Schwinger) field (the intensity $I \approx 10^{30} \mathrm{~W} / \mathrm{cm}^{2}$ ). For example, in [6], $5 \times 10^{9}$ electrons with the Lorentz factor $\gamma \approx 10^{5}$ produce only 106 electron-positron pairs for 22000 laser pulses with the intensity $I \approx 10^{18} \mathrm{~W} / \mathrm{cm}^{2}$. Besides, we completely neglect the interaction of electrons in the bunch and suppose that the size of electron's wave packet in the momentary comoving frame is of the order of several dozens of the Compton wavelengths. This does not mean that the electron bunch must be of that size, but the one-particle electron wavefunctions describing its state should be localized to this scale. Then it can be shown (see, e.g., $[7,8]$ ) that the electron wave packet center moves along a trajectory obeying the classical equations of motion (the Lorentz equation) with the electromagnetic field representing a superposition of the external field and the field created by an electron wave packet. If the wave packet is localized enough, the standard arguments used in deriving the LorentzDirac (LD) equation (see, e.g., [9-16]) transform this 
Lorentz equation into the LD equation in the leading nontrivial order in the size of the wave packet. So, in fact, we have to investigate the solutions to the LD equation. This equation is the leading order approximation to the evolutionary equation for the average electron coordinate in the in-in formalism (see, e.g., [8]). In the standard $S$ matrix approach, we need to sum an infinite number of diagrams with soft photons in order to describe such dynamics of the electron wave packet.

The quantum corrections due to radiation of hard photons can be approximately taken into account introducing an additional stochastic force to the equations of motion of the wave packet center [17]. This results in a broadening of the wave packet, but the dynamics of its center are still described by the classical equations of motion under the assumptions made. Usually, the relevance of quantum effects is characterized by the parameter $\chi$ of [2]: if $\chi \geq 1$, the quantum effects are relevant. This estimation is based on a onevertex amplitude with the initial Volkov state for the electron. The Volkov states are not localized in space. So these estimations are not immediately applicable to the case when the electrons are described by localized wave-packets and/or the radiation process becomes essentially non-perturbative. Nevertheless, on dimensional grounds, one may expect that for $\chi \gg 1$ the quantum corrections become relevant. However, as discussed thoroughly in [18], the electrons in a strong electromagnetic wave are rapidly de-excited to the state with a minimum radiation (the late time asymptotic expressions), i.e., $\chi$ becomes small since the electrons will move along the electromagnetic wave with the velocity close to the speed of light. The late time asymptotic expressions, we shall use in analyzing the properties of the scattered electrons, are attractors for the set of physical solutions to the LD equation [19, 18]. Having emitted a hard photon and suffered a recoil, the electron tends to return to the initial trajectory. The correction to the LD equation due to spin can be also neglected for the unpolarized wave packets of electrons with a vanishing average spin. The effect of radiative polarization is negligibly small in the ultrarelativistic case we consider (see [20] and for a recent discussion, e.g., [21]).

So, if we apply the LD equation to describe the electron dynamics in the field of a plane linearly polarized electromagnetic wave with the intensity $I \gtrsim$ $10^{24} \mathrm{~W} / \mathrm{cm}^{2}$ and the energy of photons $\Omega \approx 1 \mathrm{eV}$, we shall see that, in the ultrarelativistic limit $\gamma \geqslant 10^{2}$, the electrons scattered by this wave can cross the laser beam or be reflected from it. We consider a planar problem when the electron bunch moves in the polarization plane of the electromagnetic wave. A general problem can be reduced to this one by an appropriate Lorentz transform [18]. In the initial frame, the problem is essentially planar so long as the momentum component normal to the polarization plane is much lesser than the component lying in it. In the case of a planar motion, the momentum of an electron passed the electromagnetic wave depends weakly on the initial momentum and is determined by the laser beam parameters and the phase of electromagnetic wave at the electron entrance point. In particular, such electrons possess the identical momentum projections to the axis normal to the laser beam. This projection is specified solely by the laser beam diameter measured in the classical electron radii. As for the reflected electrons, there is a reflection law that relates the reflection and incidence angles. This law is independent of any parameters. The penetration depth of the reflected electrons to the laser beam is much smaller than the wavelength of the electromagnetic wave. Of course, these results are valid in a certain approximation that will be described in detail.

Practically speaking, we study the properties of the exact solutions of the so-called Landau-Lifshitz (LL) equation [22] since the exact physical solutions of the LD equation cannot be found for the field configurations we discuss. It was shown in [18] that, for such field configurations, the solutions of the LL equation provide a good approximation to the physical solutions of the LD equation even for the strong field strengths. Moreover, the solutions of the LL and LD equations tend asymptotically to each other at large times. The LL equation now becomes a standard tool in describing the evolution of electrons in a laser field with radiation reaction taken into account (see, e.g., [23-29]). The results of these papers also demonstrate that the behavior of electron's dynamics changes qualitatively for large field strengths of the laser wave. However, these investigations are mostly numerical and cannot give a clear insight what happens in this radiation dominated regime. In many of these papers, to simplify the numerical simulations, some terms of the LL equation are thrown away (see, e.g., $[30,31]$ ) without a rigorous justification, or even one dimensional simulations are only given (see, e.g., [32, 33]). Therefore, it is of importance to analyze the exact solutions in the case when they can be found. Note that the exact solutions we shall study do not display the radiation reaction trapping effect [30,31]. Though there is a considerable part of the electrons that escape the laser beam and move at small angles (less than $2^{\circ}$ for the intensities $I \geqslant 10^{25} \mathrm{~W} / \mathrm{cm}^{2}$ ) to the laser beam propagation direction. Another point is that certain regions (we shall accurately describe them) of the strong laser beam do become opaque for the ultrarelativistic electrons, as reported in [31, 34], however other regions of the beam transmit the electrons.

We use the same notation as in [22]. Besides, we put the speed of light $c=1$ and measure all the lengths in the Compton wavelengths $l_{C}=\hbar / m c$. Then the LL equation becomes

$\dot{V}_{\mu}=f_{\mu v} V^{v}+\lambda\left(\dot{f}_{\mu v} V^{v}+f_{\mu v} f_{V_{\rho}}^{v \rho}-v^{\lambda} f_{\lambda v} f_{V_{\rho} V_{\mu}}^{v \rho}\right),(1)$ 
where $\lambda=2 e^{2} /(3 \hbar)$ and $v_{\mu}=\dot{x}_{\mu}$ is the 4-velocity. The dimensionless electromagnetic fields $f_{\mu \nu}=$ $\operatorname{sgn}(e) F_{\mu v} / E_{0}$ have been introduced, where

$$
E_{0}=\frac{m^{2}}{|e| \hbar} \approx 4.41 \times 10^{13} \mathrm{G}=1.32 \times 10^{16} \mathrm{~V} / \mathrm{cm}
$$

The energies are measured in the electron rest energies $m \approx 0.511 \mathrm{MeV}$ and so the 4-velocity coincides with the 4-momentum. The modern accelerator facilities are able to accelerate electrons up to $50 \mathrm{GeV}$. The intensities of the laser fields accessible at the present moment $[27,35]$ are of the order $10^{22} \mathrm{~W} / \mathrm{cm}^{2}$ with the photon energies about $1 \mathrm{eV}$. Thus

$$
\gamma \approx 10^{5}, \quad \omega_{0} \approx 1.47 \times 10^{-4}, \quad \Omega \approx 1.96 \times 10^{-6},
$$

where $\gamma$ is the Lorentz factor, $\omega_{0}$ is the electromagnetic field strength in a laser beam, and $\Omega$ is the photon energy. We suppose that 11 is given by (3) throughout this paper.

\section{DYNAMICS OF ELECTRONS IN A PLANE WAVE}

Let us consider a monochromatic linearly polarized laser beam with the energy of photons $\Omega$ propagating along the $y$ axis. We can simulate such a laser beam by a plane electromagnetic wave

$$
\begin{gathered}
f^{\mu v}=\omega\left(x_{-}\right) \mathrm{e}_{-}^{[\mu} \mathrm{e}_{1}^{v]}, \quad f_{\mu v}^{2}=\omega^{2} \mathrm{e}_{\mu}^{-} \mathrm{e}_{v}^{-}, \\
e_{x}=-\omega, \quad h_{z}=\omega,
\end{gathered}
$$

where $\omega\left(x_{-}\right)=\omega_{m} \cos \psi, \psi=\Omega x_{-}+\psi_{0}, x_{-}=x^{0}-y$, and $\psi_{0}$ is a constant phase. The 4 -vectors $\mathrm{e}_{-}^{\mu}=(1,0,1,0)$ and $\mathrm{e}_{1}^{\mu}=(0,1,0,0)$ are also introduced. Suppose that this electromagnetic field vanishes outside the strip $x \in$ $[0, d]$, where $d$ is the laser beam diameter. This is a certain approximation, of course, to a real situation (for the description of real laser beams see, e.g., [5]). The photons in the wave packet of the width $d$ must possess the transverse momentum of the order of $2 \pi / d$. If $d \geqq$ $5 \lambda_{\gamma}$, where $\lambda_{\gamma}=2 \pi / \Omega$ is the photon wavelength, then the relative correction to the photon energy coming from the transverse momentum amounts only $k_{\perp}^{2} /\left(2 \Omega^{2}\right) \lesssim 1 / 50$, and so the expression (4) is a good approximation to the real wave packet of the width $d$. We shall assume that $\mathrm{d}$ is of the order of $5 \lambda_{\gamma}$. However, as will be shown, all the results we obtain depend weakly on the beam diameter (as $d^{1 / 3}$ ).

As for the electrons, we suppose they move in the plane $z=0$ in the $x$ axis direction and strike the laser beam at the angle $\varphi$ counted from the $y$ axis. The exact solutions to the LD equation cannot be found in this case, but the LL equation is integrable. The solution to the LL equation has the form (see [36] and references there, and also [18, 25, 37, 38])

$$
\begin{aligned}
V_{-}= & {\left[V_{-}^{-1}(0)+\frac{\lambda \omega_{m}^{2}}{4 \Omega}\left(2 \Omega x_{-}+\sin 2 \psi-\sin 2 \psi_{0}\right)\right]^{-1}, } \\
x_{-}\left[V_{-}^{-1}(0)-\frac{\lambda \omega_{m}^{2}}{4 \Omega} \sin 2 \psi_{0}\right] & +\frac{\lambda \omega_{m}^{2}}{4 \Omega^{2}}\left(\Omega^{2} x_{-}^{2}+\sin ^{2} \psi-\sin ^{2} \psi_{0}\right)=\tau \\
r= & r(0)-\lambda \omega_{m}\left(1+\frac{\omega_{m}^{2}}{2 \Omega^{2}}\right)\left(\cos \psi-\cos \psi_{0}\right) \\
- & {\left[\frac{\omega_{m}}{\Omega V_{-}}-\frac{\lambda \omega_{m}^{3}}{4 \Omega^{2}} \sin 2 \psi_{0}\right]\left(\sin ^{2} \psi-\sin _{0}\right) } \\
& -\frac{\lambda \omega_{m}^{3}}{2 \Omega} x_{-} \sin \psi+\frac{\lambda \omega_{m}^{3}}{6 \Omega^{2}}\left(\cos ^{3} \psi-\cos ^{3} \psi_{0}\right)
\end{aligned}
$$

where $r=v_{x} / v_{-}=d x / d x_{-}, v_{-}=v^{0}-v_{y}, v^{0}=\gamma$, and we have set $x_{-}(0)=0$. Notice that the misprint was made in Eq. (55) of [18]: the last term in the expression for $r$ was missed. For $\lambda=0$ the solution (5) transforms into the well-known solution to the Lorentz equation in the plane electromagnetic wave with a linear polarization (see, e.g., [22]). If $x_{-}$is small, the solution (5) becomes

$$
\begin{gathered}
V_{-}=\left[v_{-}^{-1}(0)+\lambda \bar{\omega}^{2} x_{-}\left(1-\Omega x_{-} \tan \psi_{0}\right)\right]^{-1}, \\
x_{-} V_{-}^{-1}(0)+\frac{\lambda \bar{\omega}^{2}}{2} x_{-}^{2}\left(1-\frac{2 \Omega x_{-}}{3} \tan \psi_{0}\right)=\tau, \\
r=r(0)-\frac{\bar{\omega} x_{-}}{V_{-}(0)}\left(1-\frac{\Omega x_{-}}{2} \tan \psi_{0}\right) \\
-\frac{\lambda \bar{\omega}^{3}}{2} x_{-}^{2}\left(1-\frac{4 \Omega x_{-}}{3} \tan \psi_{0}\right)+\lambda \bar{\omega} x_{-} \tan \psi_{0},
\end{gathered}
$$

where $\bar{\omega}=\omega_{m} \cos \psi_{0}$ and only the leading correction in $\Omega x_{-}$is retained. Integrating (6), we have

$$
\begin{gathered}
x=r(0) x_{-}-\bar{\omega} x_{-}^{2}\left(1-\Omega x_{-} \tan \psi_{0} / 3\right) v_{-}^{-1}(0) / 2 \\
-\lambda \bar{\omega}^{3} x_{-}^{3}\left(1-\omega x_{-} \tan \psi_{0}\right) / 6+\lambda \bar{\omega} \Omega x_{-}^{2} \tan \psi_{0} / 2
\end{gathered}
$$

where $x(0)=0$. As for $r\left(x_{-}\right)$in (5), the integration over $x_{-}$is readily performed, but the resulting expression is rather huge and we do not write it here. The mass-shell 


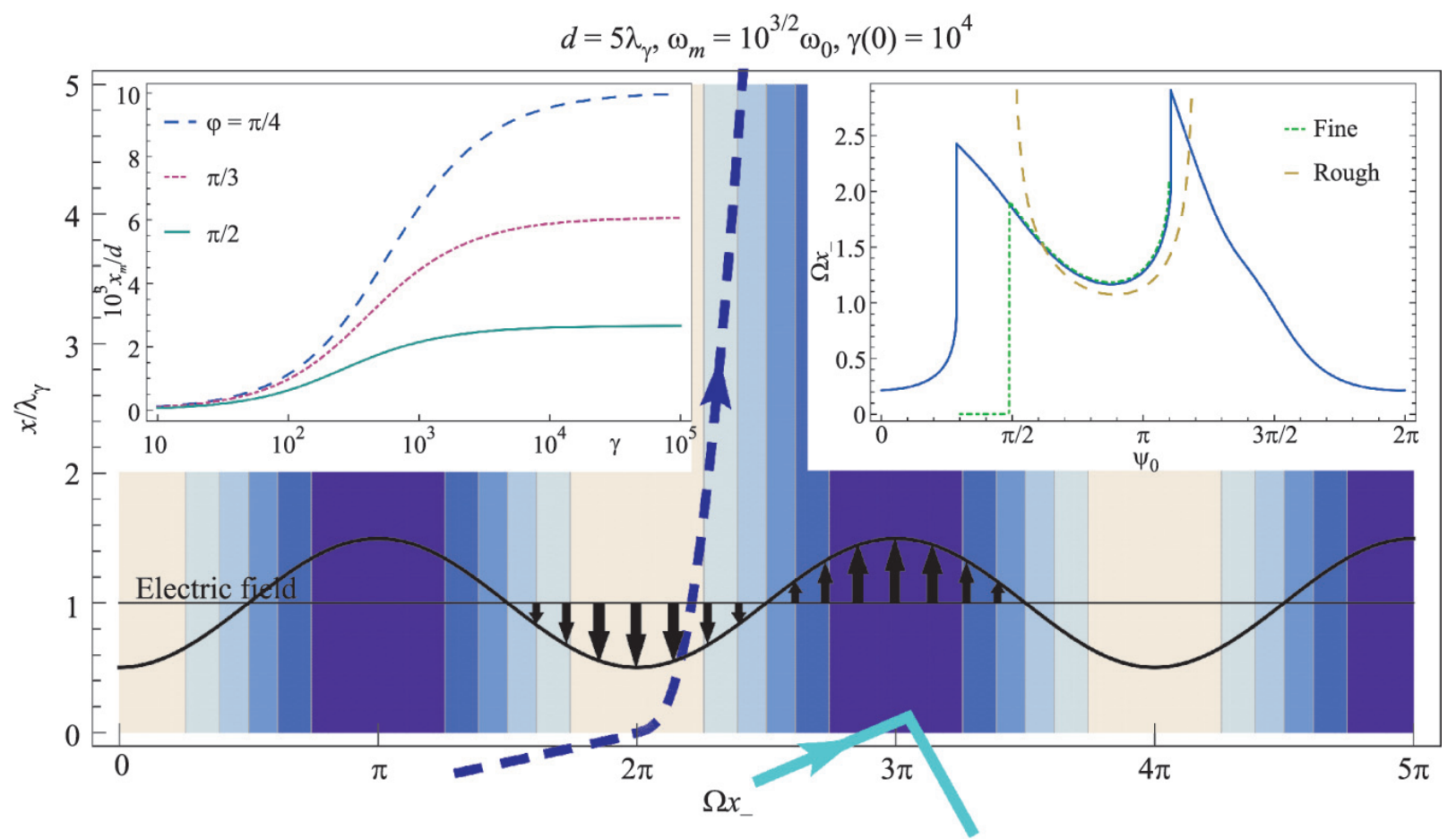

Fig. 1. (Color online) Dynamics of electron scattering by a strong electromagnetic plane wave. The sine curve shows the electric vector in the wave. The trajectory of the electron crossed the laser beam (the dashed curve) is well described by Eq. (7) without the terms depending on the initial momenta. As for the reflected electrons (the solid curve), the absolute value of the exit angle tangent is twice the incidence angle tangent (19). The penetration depth is depicted schematically. The left inset: the penetration depth in the laser beam diameters. The asymptotic behavior at large $\gamma$ values is in agreement with Eq. (18). The right inset shows the electromagnetic wave phase that the electron spends in it versus the initial phase characterizing the electron entrance point. The rough estimate is given by Eq. (11), while the fine estimate is obtained using the approximate expression for $x\left(x_{-}\right)$, where the terms depending on the initial momenta are thrown away (the case (ii) in (10)).

condition, $V^{2}=1$, implies that all the 4-momentum components are expressed in terms of $\mathrm{V}_{-}$and $r$ :

$$
\begin{gathered}
V^{0}=\frac{V_{-}}{2}\left(v_{-}^{-2}+r^{2}+1\right), \\
V_{y}=\frac{V_{-}}{2}\left(v_{-}^{-2}+r^{2}-1\right), \quad v_{x}=V_{-} r .
\end{gathered}
$$

It is convenient to express the initial momentum though the $\gamma$ factor and the entrance angle $\varphi$ of the electron to the laser beam

$$
V_{-} \approx 2 \gamma \sin ^{2}(\varphi / 2), \quad r \approx \cot (\varphi / 2), \quad \varphi \in[0, \pi],
$$

where $\gamma \gg 1$ and $\varphi \gg \gamma^{-1}$. The solutions (5) or (6) do not display the radiation reaction trapping effect [30, 31]. A secular term (the penultimate term in the expression for $r$ ) $[36,37]$ dominates at $x_{-}$large and drives the electron out of the laser beam. A similar term enters the solution for a circularly polarized electromagnetic wave (see, e.g., Eq. (52) in [18]).

\section{PROPERTIES OF THE SCATTERED ELECTRONS}

It is clear that two cases are possible: (a) the electron passes the laser beam and escapes from its oppo- site side, or (b) the electron is reflected by the electromagnetic wave. Consider, at first, the case (a).

In order to determine the momentum of the electron escaped, it is necessary to find the minimal positive root $x_{-}^{f}$ of the equation $x\left(x_{-}\right)=d$, to substitute it to the expressions (5) or (6), and then to employ formulas (8). Generally, this momentum depend on the initial data $\gamma$ and $\varphi$. However, as seen from (5), (6), if the field strength of the electromagnetic wave is large enough, $\gamma \gg 1$, and $\varphi \gg \gamma^{-1}$, the dependence on the initial data $\gamma$ and $\varphi$ becomes negligibly small. The main contribution to the final momentum comes from the terms that are proportional to $\lambda$, i.e., from the terms that arise due to radiation reaction. The dependence on $V_{-}(0)$ and $r(0)$ is negligible provided that

$$
\begin{gathered}
\text { (i) } \lambda \bar{\omega}^{2} x_{-} v_{-}(0) \gg 1, \quad r(0) \ll \lambda \bar{\omega}^{3} x_{-}^{2} \\
\text { for } \Omega x_{-} \ll 1, \\
\text { (ii) } \lambda \omega_{m}^{2} x_{-} v_{-}(0) \gg 1, \quad \Omega r(0) \ll \lambda \omega_{m}^{3} x_{-}^{2}
\end{gathered}
$$$$
\text { for } \Omega x_{-} \geq 1 \text {. }
$$

Simple analytical formulas for the momenta of escaping electrons can be obtained in the case (i) only. It is this case that is realized for the strong fields and large

\section{JETP LETTERS Vol. $101 \quad$ No. 22015}


$\gamma$, since it takes a small part of the electromagnetic wave period for a charged particle to traverse the laser beam (see Fig. 1). This, in particular, implies that the assumption of a laser wave monochromaticity is not relevant in the case (i).

From (7) in the case (i), we have

$$
\begin{gathered}
x_{-}^{f} \approx-\bar{\omega}^{-1}\left(\frac{6 d}{\lambda}\right)^{1 / 3}(1+\varepsilon)^{-1 / 3}, \\
\varepsilon=\frac{\Omega}{\bar{\omega}}\left(\frac{6 d}{\lambda}\right)^{1 / 3} \tan \psi_{0} .
\end{gathered}
$$

The approximate equality is justified in the case when $|\varepsilon| \lesssim 1$ and $\lambda \bar{\omega} / d \ll 1$. The latter inequality is satisfied for $d \gtrsim 5 \lambda_{\gamma}$ and reasonable $\omega_{m}$ values. In general, the last term in (6), (7) can be neglected in comparison with the penultimate term in these expressions. Henceforward, we omit these small terms. Formula (11) is valid only for the initial phases $\cos \psi_{0}<0$ such that the electron hits the region of the electromagnetic wave where the $E_{x}$ is negative (before the redefinition (1)), i.e., the field assists the electron to cross the laser beam, see Fig. 1 . Remark that $|\varepsilon| \lesssim 1$ for the strengths $\omega_{m} \geq 10^{3 / 2} \omega_{0}$ at $d \sim 5 \lambda_{\gamma}$. Besides, the value of $\psi_{0}$ must be sufficiently far from the points $\pi / 2+\pi n$, $n \in \mathbb{Z}$.

Making use of (11) and (6), we arrive at the approximate expressions

$$
\begin{gathered}
V_{x}^{f} \approx \frac{1}{2}\left(\frac{6 d}{\lambda}\right)^{1 / 3} \frac{1+4 \varepsilon / 3}{(1+\varepsilon)^{4 / 3}}, \quad V_{-}^{f} \approx-\frac{(\lambda / 6 d)^{1 / 3}}{\lambda \bar{\omega}(1+\varepsilon)^{2 / 3}}, \\
v_{-}^{f} V_{x}^{f} \approx-\frac{1+4 \varepsilon / 3}{2 \lambda \bar{\omega}(1+\varepsilon)^{2}}, \quad V_{y}^{f} \approx-\frac{3 \bar{\omega} d(1+4 \varepsilon / 3)^{2}}{4(1+\varepsilon)^{2}}, \\
V_{0}^{f} \approx v_{y}^{f}, \quad \tan \alpha \approx-\frac{2(6 d / \lambda)^{1 / 3}}{3 \bar{\omega} d} \frac{(1+\varepsilon)^{2 / 3}}{1+4 \varepsilon / 3},
\end{gathered}
$$

where $\alpha$ is the electron exit angle counted from the $y$ axis. The relations (12) are valid so long as the conditions (10) for the case (i) are fulfilled. These conditions are written as

$$
\begin{gathered}
\lambda \bar{\omega} \gamma \sin ^{2} \frac{\varphi}{2}\left(\frac{6 d}{\lambda}\right)^{1 / 3} \gg 1, \quad \cot \frac{\varphi}{2} \ll \frac{\lambda \bar{\omega}}{4}\left(\frac{6 d}{\lambda}\right)^{2 / 3}, \\
(6 d / \lambda)^{1 / 3} \ll-\bar{\omega} / \Omega,
\end{gathered}
$$

respectively. Numerical simulations show that the restrictions above can be weaken to a large extent, and formulas (12) still hold with a high accuracy (see Fig. 2). As follows from the general solution (see Eq. (55) of [18]) in the non-planar case, when $v_{z} \neq 0$, the first three formulas in (12) do not change. The other expressions in (12) remain valid so long as

$$
\frac{v_{z}^{2}(0)}{V_{-}^{2}(0)} \ll r_{f}^{2} \approx \frac{\lambda^{2} \bar{\omega}^{2}}{4}\left(\frac{6 d}{\lambda}\right)^{4 / 3} \text {. }
$$

As mentioned in the Introduction, the electrons are rapidly de-excited in the electromagnetic wave, and the parameter $\chi:=|\bar{\omega}| v_{-}$characterizing a relevance of quantum corrections becomes small. Indeed, from (12) we have

$$
\chi \approx \frac{(\lambda / 6 d)^{1 / 3}}{\lambda(1+\varepsilon)^{2 / 3}} \approx 0.076,
$$

where we have put $\varepsilon=0$ and taken $d=5 \lambda_{\gamma}$ in the last approximate equality. In the leading order in $\varepsilon$, the final value of $\chi$ is independent of the initial Lorentz factor and the field strength of the electromagnetic wave.

Notice also that the mistake was made in formula (121) of [18] and in the estimations following from it since this formula does not take into account the essentially nonlinear dynamics of the electron in the asymptotic regime. In particular, the proper-time of the electron, when it escapes from the laser beam, is equal to

$$
\tau_{\mathrm{esc}} \approx \frac{\lambda}{2}\left(\frac{6 d}{\lambda}\right)^{2 / 3} \frac{1+2 \varepsilon / 3}{(1+\varepsilon)^{2 / 3}} .
$$

The initial data are assumed to satisfy the conditions (13). The restriction on the angles (126) of [18], where the radiation formed on the asymptotics can be observed, becomes

$$
\begin{gathered}
|\zeta|=\left(\frac{k_{1}^{2}+k_{3}^{2}}{k_{-}^{2}}\right)^{1 / 2} \\
=\cot \frac{\delta}{2} \ll \frac{\lambda|\bar{\omega}|}{2}\left(\frac{6 d}{\lambda}\right)^{2 / 3} \frac{1+2 \varepsilon / 3}{(1+\varepsilon)^{2 / 3}} .
\end{gathered}
$$

Here $\delta$ is the exit angle of a photon counted from the $y$ axis. For $\varepsilon=0, d=5 \lambda_{\gamma}$, and $\bar{\omega}=10^{3 / 2} \omega_{0}$, the quantity standing on the right-hand side of the inequality is approximately 82.7 . In the leading order in e, the spectral density of radiation in the case we consider is described by the formulas presented in Section 5.1 of [18].

Up to the terms of the order of $\varepsilon^{2}$, the momentum component $v_{x}^{f}$ of the electrons crossed the laser beam is determined solely by the laser beam diameter $d$ measured in the classical electron radii. A numerical analysis reveals that the rough estimate $(\varepsilon=0)$ for $v_{x}^{f}$ is satisfied with the accuracy of $1.4 \%$ for almost all the ultrarelativistic electrons crossed the laser beam (see Fig. 2). This relation holds far beyond the validity bounds (13) of the approximations made in deriving (12). In contrast to $v_{x}^{f}$, the product $v_{-}^{f} V_{x}^{f}$ is independent of the beam diameter $d$ in the leading order in $\varepsilon$ and determined by the field strength at the entrance point of the electron to the wave [19]. 


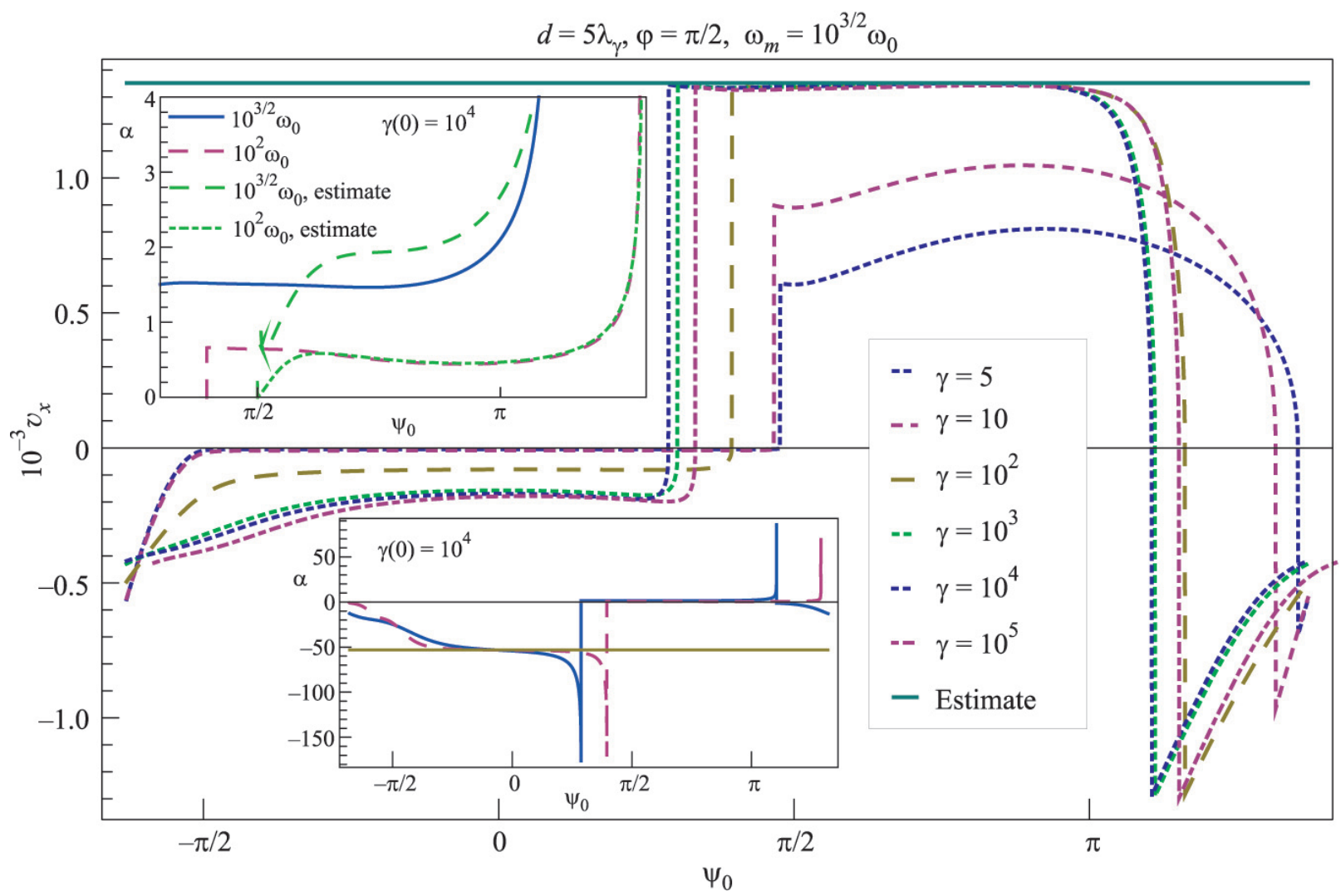

Fig. 2. (Color online) Quantity $v_{x}^{f}$ versus the initial phase. The straight line on the top is the rough estimate given by Eq. (12) at $\varepsilon=0$. Estimates (24) and (25) for zeros of $v_{x}^{f}$ are $\psi_{0}^{r} \approx 1.11 \pi, \psi_{0}^{l} \approx 0.30 \pi$, respectively. The bottom inset shows the exit angle versus the initial phase. Transmitted electrons escape a laser beam mostly at nearly equal small angles $\alpha$. The rough estimate for this angle is given by Eq. (12) at $\psi_{0}=\pi$. Reflected electrons also mostly exit at a given angle determined by the reflection law (19). The estimate following from this law is depicted as a straight line. The top inset shows comparison with the estimates (the green lines) obtained from Eq. (5) by discarding the terms depending on the initial momenta and taking $x_{-}^{f}$ from (11).

In the case (ii) of (10), the momenta of the scattered electrons are independent of the initial values $\gamma$ and $\varphi$ and specified by formulas (5), where the terms proportional to $r(0)$ or $v_{-}^{-1}(0)$ are to be omitted. The major obstacle for the analytical study in this case is the impossibility to solve the equation $x\left(x_{-}\right)=d$ in an explicit form, although it can be simply solved numerically. If $\Omega x_{-}^{f} \approx 1$, formula (11) can be used to estimate the value of $x_{-}^{f}$. The results of numerical simulations (Fig. 1) show that the latter approximation is good enough for the field strengths $\omega_{m} \geq 10^{3 / 2} \omega_{0}$ at $d \sim 5 \lambda_{\gamma}$.

Now we consider the case (b). In this case, the electron spends a short time in the laser beam. Hence, the ratio $r^{f}$ depends on $r(0)$, while the dependence of $r^{f}$ and $v_{-}^{f}$ on $V_{-}(0)$ is negligibly small in the ultrarelativistic limit. The quantity $\Omega x_{-}^{f} \ll 1$ for almost all the reflected electrons, and so one can use (7) putting $\Omega=$
0 and discarding the terms proportional to $V_{-}^{-1}(0)$ there. Then we obtain

$$
x_{-}^{f}=\sqrt{6 r(0) /\left(\lambda \bar{\omega}^{3}\right)}, \quad x_{m}=\sqrt{8 r^{3}(0) /\left(9 \lambda \bar{\omega}^{3}\right)},
$$

where $x_{m}$ is the penetration depth. Formula (18) takes place at $\cos \psi_{0}>0$ only. The penetration depth to the laser beam does not depend on the energy of the ultrarelativistic electron striking it, but is determined only by the field strength at the electron entrance point and by the incidence angle (see Fig. 1). Substituting $x_{-}^{f}$ into (6) and discarding the terms specified above, we come to

$$
r^{f}=-2 r(0), \quad v_{-}^{f}=[6 \lambda \bar{\omega} r(0)]^{-1 / 2},
$$

$$
\begin{aligned}
& v_{x}^{f}=-[2 r(0) /(3 \lambda \bar{\omega})]^{1 / 2}, \quad v_{-}^{f} v_{x}^{f}=-(3 \lambda \bar{\omega})^{-1} \text {, } \\
& v_{y}^{f} \approx \frac{4 r^{2}(0)-1}{\sqrt{24 \lambda \bar{\omega} r(0)}}, \quad v_{0}^{f} \approx \frac{4 r^{2}(0)+1}{\sqrt{24 \lambda \bar{\omega} r(0)}}, \\
& \tan \alpha=\frac{4 r(0)}{1-6 \lambda \bar{\omega} r(0)-4 r^{2}(0)} \approx \frac{4 \cot (\varphi / 2)}{1-4 \cot ^{2}(\varphi / 2)},
\end{aligned}
$$

\section{JETP LETTERS Vol. $101 \quad$ No. 22015}



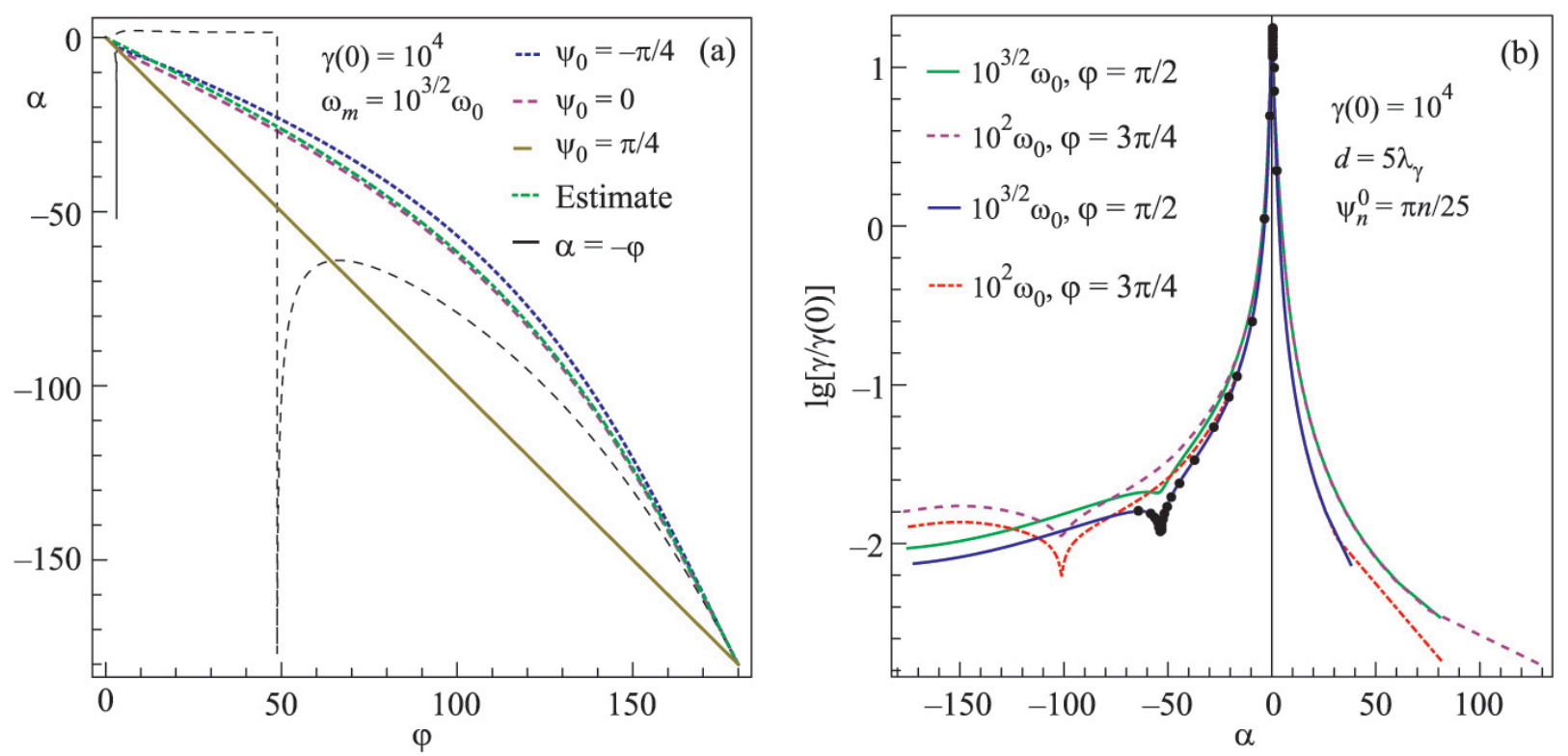

Fig. 3. (Color online) Left panel: the reflection law. Formula (19) provides a faithful estimate for the reflection angle when the phase $\psi_{0}$ lies near the plateau of the plot $\alpha\left(\psi_{0}\right)$ given in Fig. 2 . In that case, the relative error between the exit angle deduced from the exact solution and formula (19) is less than $2 \%$. The point $\psi_{0}=\pi / 4$ is not on this plateau and so a large deviation from Eq. (19) arises. Right panel: the escaping electron $\gamma$ factor versus the exit angle. The cusps on the curves at $\alpha<0$ correspond to the reflection angles prescribed by (19). The cusp at $\alpha=0$ is formed mainly by the transmitted electrons with the $\gamma$ factor approximated by (12) at $\psi_{0}=\pi$. For equiprobable initial phases, a high density of blobs on a curve corresponds to a high probability to find electrons with such an exit angle and $\gamma$ factor.

where it is supposed that $r(0) \gg \lambda \bar{\omega}$ and $\varphi \gg \gamma^{-1}$ in the approximate equalities. The expressions (19) hold when

$$
\begin{gathered}
\Omega \ll \sqrt{\lambda \bar{\omega}^{3} \tan (\varphi / 2) / 6}, \quad 8 \cot ^{3}(\varphi / 2)<9 \lambda \bar{\omega}^{3} d^{2}, \\
{\left[6 \lambda \bar{\omega} \sin ^{3}(\varphi / 2) \cos (\varphi / 2)\right]^{1 / 2} \gg \gamma^{-1} .}
\end{gathered}
$$

The first restriction is satisfied for $\cot (\varphi / 2) \lesssim 1$ and $\bar{\omega} \geq 10^{3 / 2} \omega_{0}$. The second inequality guaranteeing that the electron does not escape from the opposite side of the laser beam follows from the first one at $\cot (\varphi / 2) \lessgtr$ 1 and $d \gtrsim \lambda_{\gamma}$. The last inequality is fulfilled at $\gamma \gtrsim 10^{3}$, $\bar{\omega}=\omega_{0}$, and $\sin \varphi \gtrsim 1 / 10$. It is clear that the larger $\bar{\omega}$ and $\gamma$, the larger interval of the angles $\varphi$ complies with the restrictions (20). The parameter $\mathrm{x}$ is also small. For $\bar{\omega}=10^{3 / 2} \omega_{0}$ and $\cot (\varphi / 2) \sim 1$, we have from (19)

$$
\chi \approx\{\bar{\omega} /(6 \lambda r(0))\}^{1 / 2} \approx 0.40 .
$$

The first four formulas in (19) are left unchanged for a non-planar scattering. The other relations still hold provided

$$
v_{z}^{2}(0) / v_{-}^{2}(0) \ll r_{f}^{2} \approx 4 r^{2}(0) .
$$

The relations (19) imply that the electron is reflected from the laser beam at the angle which is determined, with a high accuracy, only by the incidence angle and not equal to it (see Fig. 3). The elec- tromagnetic wave transfers a momentum along the $\mathrm{y}$ axis to the electron, and so the absolute value of the reflection angle is always less than the incidence angle. Since

$$
\alpha^{\prime}(\varphi)=-4(3 \cos \varphi+5)^{-1},
$$

the laser wave field reflecting electrons collimates the electron bunch at the incidence angles $\varphi \in\left[0, \varphi_{0}\right]$, $\varphi_{0}=\arccos (-1 / 3) \approx 109.5^{\circ}$, i.e., being reflected, the electron bunch possesses a less dispersion of angles. For $\varphi>\varphi_{0}$, the angle dispersion in the reflected electron bunch is bigger than in the initial bunch. Similarly to the electrons crossed the laser beam, the product $V_{-}^{f} V_{x}^{f}$ for the reflected electrons is independent of the initial momentum, but equal to a different value than in (12).

It is clearly seen in Fig. 2 that the values of $\psi_{0}$ separating the region of phases $\psi_{0}$, where the electrons pass through the laser beam, from the region of phases, where the electrons are reflected, are not exactly $\psi_{0}=$ $\pi / 2+\pi n$, as one may expect from the approximate analytic formulas (11), (18). The value of the phase $\psi_{0}$, where, in increasing $\psi_{0}$, the transmission region is superseded by the reflection region, can be roughly estimated using the expression (12) for $v_{x}^{f}$. This 
momentum component vanishes at $\varepsilon=-4 / 3$. It approximately corresponds to

$$
\psi_{0} \approx \frac{3 \pi}{2}-\sqrt{\frac{4 \Omega}{3 \omega_{m}}\left(\frac{6 d}{\lambda}\right)^{1 / 3}}+2 \pi n .
$$

A similar analysis for the value of the phase $\psi_{0}$, where the reflection is superseded by the transmission, leads to

$$
\psi_{0} \approx \frac{\pi}{2}-\left[11.8 \frac{\Omega^{2} r(0)}{\lambda \omega_{m}^{3}}\right]^{1 / 5}+2 \pi n
$$

In order to obtain this formula, one needs to consider the dynamics of reflected particles and find such a value of the phase that $v_{x}^{f}=0$.

\section{CONCLUSIONS}

In sum, we obtained the relations (12), (19) for the momenta of electrons scattered by a laser beam that are quite unexpected or may be even counterintuitive. For example, the electron cannot break through some regions of the laser beam whatever high the electron energy is. For a head-on collision this fact is known for a long time (see, e.g., [18, 37] and for a non-monochromatic wave [39]), but here we described analytically such a reflection for the electrons hitting the electromagnetic wave at large angles. A simple reflection law was established. The electrons crossed the laser beam are mostly scattered at small angles to its propagation direction, the momentum component parallel to the electric vector being determined by the laser beam diameter only. The results of our semiclassical analysis can be also used to provide a clear-cut distinction between the classical and quantum effects on the dynamics of localized electron wave packets.

We are grateful to Prof. V.G. Bagrov for fruitful conversations. This work was supported by the Tomsk State University Competitiveness Program, by the Russian Foundation for Basic Research (project nos. 13-02-00551 and 12-02-01314-a), by the Council of the President of the Russian Federation for Support of Young Scientists and Leading Scientific Schools (project no. no. MK-237.2013.2), and by the Ministry of Education and Science of the Russian Federation (contract no. 1.676.2014/K, program "Nauka").

\section{REFERENCES}

1. V. B. Berestetskii, E. M. Lifshitz, and L. P. Pitaevskii, Quantum Electrodynamics (Pergamon, Oxford, 1982).

2. V. I. Ritus, J. Sov. Laser Res. 6, 497 (1985).

3. www.eli-laser.eu.

4. www.xcels.iapras.ru.

5. E. Hemsing, G. Stupakov, D. Xiang, and A. Zholents, Rev. Mod. Phys. 86, 897 (2014).
6. C. Bamber, S. J. Boege, T. Koffas, T. Kotseroglou, A. C. Melissinos, D. D. Meyerhofer, D. A. Reis, W. Ragg, C. Bula, K. T. McDonald, E. J. Prebys, D. L. Burke, R. C. Field, G. Horton-Smith, J. E. Spencer, D. Walz, S. C. Berridge, W. M. Bugg, K. Shmakov, and A. W. Weidemann, Phys. Rev. D 60, 092004 (1999).

7. V. G. Bagrov, V. V. Belov, and A. Yu. Trifonov, Ann. Phys. (N.Y.) 246, 231 (1996); arXiv:quant-ph/9806017; Methods of Mathematical Physics: Asymptotic Methods in Relativistic Quantum Mechanics (Tomsk. Politekh. Univ., Tomsk (2006) [in Russian].

8. V. Krivitskii and V. Tsytovich, Sov. Phys. Usp. 34, 250 (1991); Ph. R. Johnson and B. L. Hu, Phys. Rev. D 65, 065015 (2002).

9. H. A. Lorentz, Theory of Electrons (B. G. Teubner, Leipzig, 1909).

10. P. A. M. Dirac, Proc. R. Soc. London A 167, 148 (1938).

11. A. O. Barut, Electrodynamics and Classical Theory of Fields and Particles (Dover, New York, 1964).

12. F. Rohrlich, Classical Charged Particles (Addison-Wesley, Reading, MA, 1965).

13. N. P. Klepikov, Sov. Phys. Usp. 28, 506 (1985).

14. H. Spohn, Dynamics of Charged Particles and Their Radiation Field (Cambridge Univ. Press, Cambridge, 2004).

15. B. P. Kosyakov, Theor. Math. Phys. 199, 493 (1999); P. O. Kazinski, S. L. Lyakhovich, and A. A. Sharapov, Phys. Rev. D 66, 025017 (2002).

16. P. O. Kazinski and A. A. Sharapov, Theor. Math. Phys. 143, 798 (2005).

17. A. A. Sokolov and I. M. Ternov, Radiation from Relativistic Electrons (American Institute of Physics, New York, 1986).

18. P. O. Kazinski, Ann. Phys. (Amsterdam) 339, 430 (2013).

19. P. O. Kazinski and M. A. Shipulya, Phys. Rev. E 83, 066606 (2011).

20. I. M. Ternov, V. G. Bagrov, and A. M. Khapaev, Ann. Phys. (Leipzig) 477, 25 (1968).

21. D. V. Karlovets, Phys. Rev. A 84, 062116 (2011).

22. L. D. Landau and E. M. Lifshitz, The Classical Theory of Fields (Pergamon, Oxford, 1962).

23. F. V. Hartemann, D. J. Gibson, and A. K. Kerman, Phys. Rev. E 72, 026502 (2005).

24. A. di Piazza, K. Z. Hatsagortsyan, and C. H. Keitel, Phys. Rev. Lett. 102, 254802 (2009).

25. Y. Hadad, L. Labun, J. Rafelski, N. Elkina, C. Klier, and H. Ruhl, Phys. Rev. D 82, 096012 (2010).

26. C. Harvey, T. Heinzl, and M. Marklund, Phys. Rev. D 84, 116005 (2011).

27. A. di Piazza, C. Müller, K. Z. Hatsagortsyan, and C. H. Keitel, Rev. Mod. Phys. 84, 1177 (2012).

28. T. Schlegel and V. T. Tikhonchuk, New J. Phys. 14, 073034 (2012).

29. A. Gonoskov, A. Bashinov, I. Gonoskov, C. Harvey, A. Ilderton, A. Kim, M. Marklund, G. Mourou, and A. Sergeev, Phys. Rev. Lett. 113, 014801 (2014).

30. L. L. Ji, A. Pukhov, I. Yu. Kostyukov, B. F. Shen, and K. Akli, Phys. Rev. Lett. 112, 145003 (2014). 
31. A. M. Fedotov, N. V. Elkina, E. G. Gelfer, N. B. Narozhny, and H. Ruhl, arXiv:1408.0362.

32. A. Zhidkov, J. Koga, A. Sasaki, and M. Uesaka, Phys. Rev. Lett. 88, 185002 (2002).

33. N. Neitz and A. di Piazza, Phys. Rev. Lett. 111, 054802 (2013).

34. N. V. Elkina, A. M. Fedotov, C. Herzing, and H. Ruhl, Phys. Rev. E 89, 053315 (2014).

35. V. Yanovsky, V. Chvykov, G. Kalinchenko, P. Rousseau, T. Planchon, T. Matsuoka, A. Maksimchuk, J. Nees, G.
Cheriaux, G. Mourou, and K. Krushelnick, Opt. Express 16, 2109 (2008).

36. W. H. Kegel, H. Herold, H. Ruder, and R. Leinemann, Astron. Astrophys. 297, 369 (1995).

37. A. I. Nikishov, J. Exp. Theor. Phys. 83, 274 (1996).

38. A. di Piazza, Lett. Math. Phys. 83, 305 (2008).

39. M. Tamburini, C. H. Keitel, and A. Di Piazza, Phys. Rev. E 89, 021201(R) (2014). 